04,08

\title{
Моделирование атомной и электронной структуры вакансий и поливакансий кислорода в $\mathrm{ZrO}_{2}$
}

\section{(C) T.В. Перевалов}

Институт физики полупроводников им. А.В. Ржанова СО РАН, Новосибирск, Россия

Новосибирский государственный университет,

Новосибирск, Россия

E-mail: timson@isp.nsc.ru

С использованием квантово-химического моделирования атомной и электронной структуры исследуется оксид циркония в кубической, тетрагональной и моноклинной фазах с вакансиями и поливакансиями кислорода. Показано, что вакансия кислорода в $\mathrm{ZrO}_{2}$ может выступать в качестве и электронной, и дырочной ловушки. Электрон, добавленный в структуру $\mathrm{ZrO}_{2}$ с вакансией кислорода, распределяется между двумя ближайшими друг к другу атомами $\mathrm{Zr}$ и имеет характер связующей орбитали. Для каждой последующей вакансии О выгодно формирование вблизи уже существующих, причем с одним атомом $\mathrm{Zr}$ связано не более двух атомов О, подвергающихся удалению. Уровни дефектов от поливакансий кислорода распределяются в запрещенной зоне с преимущественной локализацией вблизи уровня моновакансии кислорода.

Работа выполнена при поддержке РФФИ, грант № 16-32-00119 мол_а. Моделирование осуществлялось на вычислительном кластере Института физики полупроводников им. А.В. Ржанова СО РАН.

DOI: 10.21883/FTT.2018.03.45537.03D

\section{1. Введение}

$\mathrm{ZrO}_{2}$ является high- $\kappa$ диэлектриком, перспективным для использования в устройствах энергонезависимой памяти [1]. В настоящее время $\mathrm{ZrO}_{2}$ привлекает внимание в связи с потенциальной возможностью его использования в качестве активной запоминающей среды устройств резистивной памяти (Resistive Random Access Memory — ReRAM) [2]. Физика резистивного эффекта в high- $\kappa$ диэлектриках является дискуссионной темой. Наиболее распространенной является так называемая филаментная модель резистивного эффекта, согласно которой под действием импульса тока в диэлектрическом слое формируется проводящий канал диаметром 1-10 nm. Простейшей моделью филамента является цепочка вакансий кислорода, т. е. поливакансия. Изучение возможности (энергетической выгодности) формирования цепочек вакансий кислорода в $\mathrm{ZrO}_{2}$, а также их способности участвовать в транспорте заряда представляет интерес как с практической точки зрения, так и для фундаментальной физики.

Изучение свойств вакансий кислорода в $\mathrm{ZrO}_{2}$, в частности принципиальной возможности вакансий кислорода формировать цепочки и их способности участвовать в проводимости заряда, представляет большой теоретический и практический интерес. $\mathrm{ZrO}_{2}$, используемый в ReRAM, как правило, имеет поликристаллическую структуру [3]. В этом свете интерес представляет изучение атомной и электронной структуры вакансий и поливакансий кислорода в $\mathrm{ZrO}_{2}$ в различных кристаллических фазах, которые возможно моделировать из первых принципов. Электронная структура вакансий кислорода в $\mathrm{ZrO}_{2}$ моделировалась неоднократно [4-9]. Большей частью в этих работах используется стандарт- ная теория функционала плотности на основе приближения локальной плотности (LDA) или обобщенного градиентного приближения (GGA), которая существенно недооценивает значение ширины запрещенной зоны диэлектриков [4-7]. Очевидно, такой подход не способен дать корректного положения дефектных состояний в запрещенной зоне. Кроме того, авторы ограничиваются моделированием одной из трех кристаллических фаз $\mathrm{ZrO}_{2}$ : кубической $[6,7]$, тетрагональной [9] и моноклинной $[4,5,8]$. Насколько нам известно, электронная структура поливакансий кислорода в $\mathrm{ZrO}_{2}$ не изучалась.

Вакансии и поливакансии кислорода моделировались в суперячейках из 192 атомов для $c$ - и $t-\mathrm{ZrO}_{2}$ и 96 атомов для $m-\mathrm{ZrO}_{2}$. Каждый последующий атом кислорода удалялся из суперячейки по принципу минимума энергии формирования, путем перебора всех возможных вариантов взаимного расположения вакансий. Предлагаемая модель отражает последовательный рост размера поливакансии.

Моделирование электронной структуры $\mathrm{ZrO}_{2}$ осуществлялось для кубической $F m \overline{3} m(c$-), тетрагональной $P 4_{2} / n m c(t-)$ и моноклинной $P 21 / c(m-)$ фаз. Хотя в нормальных условиях кристаллический $\mathrm{ZrO}_{2}$ стабилен лишь в моноклинной фазе, интерес к высокотемпературным $c$ - и $t$-фазам обусловливают экспериментальные данные, показывающие, что в тонких пленках $\mathrm{ZrO}_{2}$ толщиной $10-50$ нм наблюдается формирование $c$ - и $t-\mathrm{ZrO}_{2}$ при температурах около 200 и $400^{\circ} \mathrm{C}$ соответственно [10]. В $c$ - и $t$-фазах атомы $\mathrm{O}$ четырехкратно координированы $\mathrm{Zr}$, в $m-\mathrm{ZrO}_{2}$ в равном отношении трехкратно (3fold) и четырехкратно (4-fold) координированные атомы О. Расчеты осуществлялись в программном пакете Quantum ESPRESSO. 
Для суперячейки с моновакансией выбор второго атома О для удаления осуществляется перебором всех возможных вариантов и определением наименьшей энергии формирования дефекта, рассчитываемой по формуле

$$
E_{\text {form }}\left(N_{\text {vac }}\right)=E_{N \text { vac }}-E_{\text {perfect }}
$$

Здесь $E_{\text {perfect }}$ - полная энергия бездефектной суперячейки, $E_{N v a c}-$ энергия суперячейки с $N$ вакансиями, $N$ - количество удаленных атомов кислорода, $E\left(\mathrm{O}_{2}\right)$ - полная энергия молекулы кислорода в триплетном состоянии. Структура тривакансии определяется для фиксированной пары вакансий по аналогичному принципу и является отправной точкой для определения структуры тетравакансии. Предлагаемая модель отражает последовательный рост размера поливакансии. Описанная процедура является весьма ресурсозатратной: для выяснения оптимального положения четырех вакансий О в исследуемых структурах выполнено 972 расчета релаксации геометрии. Эти расчеты осуществляются в рамках стандартной теории функционала плотности (ТФП). Расчеты спектров полной и парциальной плотности состояний (TDOS и PDOS) проводятся с использованием гибридного функционала B3LYP. Это наиболее популярный и апробированный функционал, который дает близкое к экспериментальному значение ширины запрещенной зоны диэлектриков. Волновые функции валентных электронов $\mathrm{Zr} 4 d 25 s 2$ и О $2 s 22 p 4$ описываются разложением по базису из плоских волн c энергией отсечки $55 \mathrm{Ry}$, а остов учитывается через псевдопотенциалы.

Термическая $E_{\text {th }}$ и оптическая $E_{\text {opt }}$ энергии ионизации ловушки оценивались по формулам, предложенным в работе [11]:

$$
\begin{aligned}
E_{\mathrm{th}}\left(V^{q}\right) & =\left[E^{-}+E_{q+1}^{q+1}\right]-\left[E^{0}+E_{q}^{q}\right], \\
E_{\mathrm{opt}}\left(V^{q}\right) & =E_{\mathrm{th}}\left(V^{q}\right)+E_{\mathrm{relax}}\left(V^{q+1}\right) \\
& =E_{\mathrm{th}}\left(V^{q}\right)+\left[E_{q}^{q+1}-E_{q+1}^{q+1}\right] .
\end{aligned}
$$

Здесь $E_{0}$ и $E^{-}$- полные энергии нейтральной и отрицательно заряженной бездефектной суперячейки соответственно; $E_{q}^{q}$ и $E_{q+1}^{q+1}-$ энергии суперячейки с вакансией $\mathrm{O}$ в зарядовых состояниях $q$ и $q+1(q=-2$, $-1,0,+1)$ соответственно, причем верхние индексы обозначают зарядовое состояние дефекта, а нижние для какой равновесной геометрии (т.е. отвечающей какому зарядовому состоянию дефекта) осуществляется расчет; $E_{q}^{q+1}$ - полная энергия суперячейки с вакансией $\mathrm{O}$ в зарядовом состоянии $q+1$, но в геометрии, соответствующей зарядовому состоянию $q$.

\section{2. Результаты}

Расчетные значения ширины запрещенной зоны для $c$-, $t$ - и $m-\mathrm{ZrO}_{2}$ равны $5.7,6.1$ и 5.6 эВ соответственно и согласуются с экспериментальными значениями 5.1, 5.78 и $5.83 \mathrm{eV}[12]$. Энергии формирования трехкратно (3-fold) и четырехкратно (4-fold) координированной вакансии кислорода, равные 6.11 и $6.17 \mathrm{eV}$, предсказывают превалирование в реальных структурах 4-fold вакансий.

Положение дефектных уровней (состояний КонаШема) относительно $E_{c}$ и $E_{v}$, обусловленных нейтральной, однократно и двукратно отрицательно и положительно заряженной вакансией $\mathrm{O}$, представлено на рис. 1 . Данный тип расчетов ввиду трудоемкости осуществлялся только для $m-\mathrm{ZrO}_{2}$ фазы, как наиболее стабильной и структурно близкой к аморфной. При добавлении электрона в запрещенной зоне формируется второе локализованное однократно заполненное состояние на 0.8 и $1.2 \mathrm{eV}$ ниже $E_{c}$ для 3-fold и 4-fold вакансий O соответственно. Добавление второго электрона приводит к двум возможным состояниям дефекта: синглетному $\left(V^{-2}(s)\right)$ и триплетному $\left(V^{-2}(t)\right)$, причем последнее примерно на $0.06 \mathrm{eV}$ ниже по полной энергии. В случае $V^{-2}(t)$ в запрещенной зоне имеется три уровня, причем два уровня спин-поляризованы и нижний из них отстоит от края зоны проводимости на $1.2 \mathrm{eV}$ для 3-fold вакансии и на $1.28 \mathrm{eV}$ для 4-fold вакансий. Большая глубина дефектных уровней от отрицательно заряженной вакансии $\mathrm{O}$ и то, что в отсутствии дополнительного электрона эти состояния попадают в зону проводимости, говорит о значительной релаксации атомов и электронной плотности вокруг дефекта. Можно сказать, что электрон локализуется в яме, которую сам себе „вырывает“ (поляронный эффект).

Положительно заряженная вакансия кислорода (убран один электрон) также формирует дополнительный уровень в запрещенной зоне вблизи $E_{c}$, при этом состояние от нейтральной вакансии становится заполненным однократно и практически не смещается. Полностью ионизованная кислородная вакансия дает одно пустое состояние в запрещенной зоне, причем вблизи $E_{c}$.

О том, что дополнительный электрон или дырка локализованы на вакансии, говорят малые значения $(\sim 0.1 \mathrm{eV})$ ширины пиков в спектре TDOS от соответствующих дефектных состояний в запрещенной зоне. Факт локализации заряда также подтверждает картина пространственного распределения зарядовой плотности от избыточного заряда в дефектной суперячейке, представленная на рис. 2. Видно, что и положительный и отрицательный заряд локализованы, причем в области вакансии и ее первой координационной сферы. Дополнительный электрон приводит к распределению зарядовой плотности, в частности между двумя атомами $\mathrm{Zr}$, и можно сказать, что формируется своего рода $\mathrm{Zr}-\mathrm{Zr}$-связь.

Расчетные значения $E_{\text {opt }}$ и $E_{\text {th }}$ для различных зарядовых состояний вакансии кислорода в $m-\mathrm{ZrO}_{2}$ представлены в таблице. Выражение для $E_{\text {th }}$ можно также интерпретировать как выигрыш в энергии при захвате электрона со дна зоны проводимости на дефектное состояние. Положительные расчетные величины $E_{\mathrm{th}}$ указывают на то, что захват электрона на вакансию кислорода в $m-\mathrm{ZrO}_{2}$ энергетически выгоден. Таким образом, 

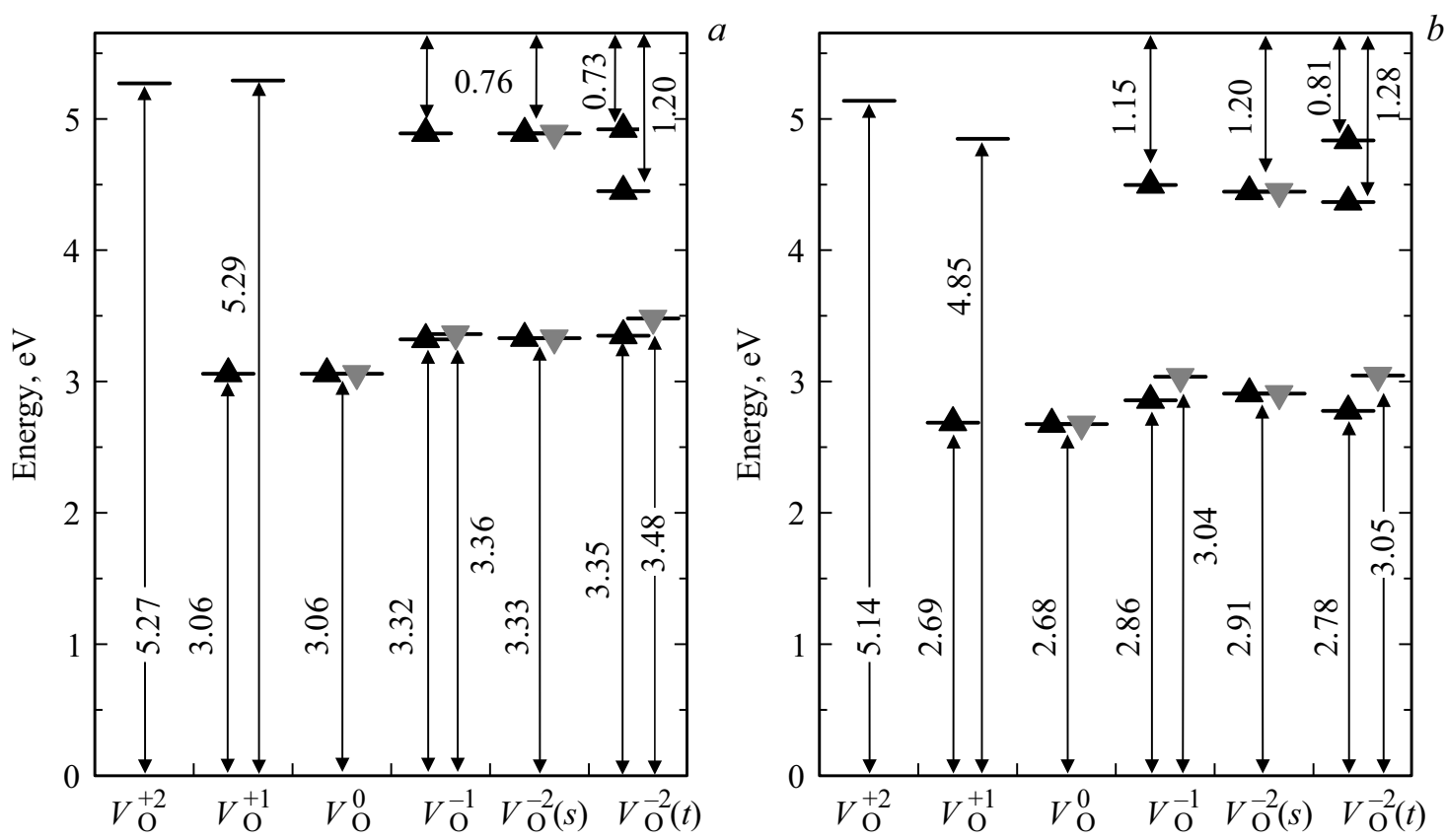

Рис. 1. Дефектные уровни в запрещенной зоне $m-\mathrm{ZrO}_{2}$ для трехкратно (a) и четырехкратно $(b)$ координированной вакансии $\mathrm{O}$.
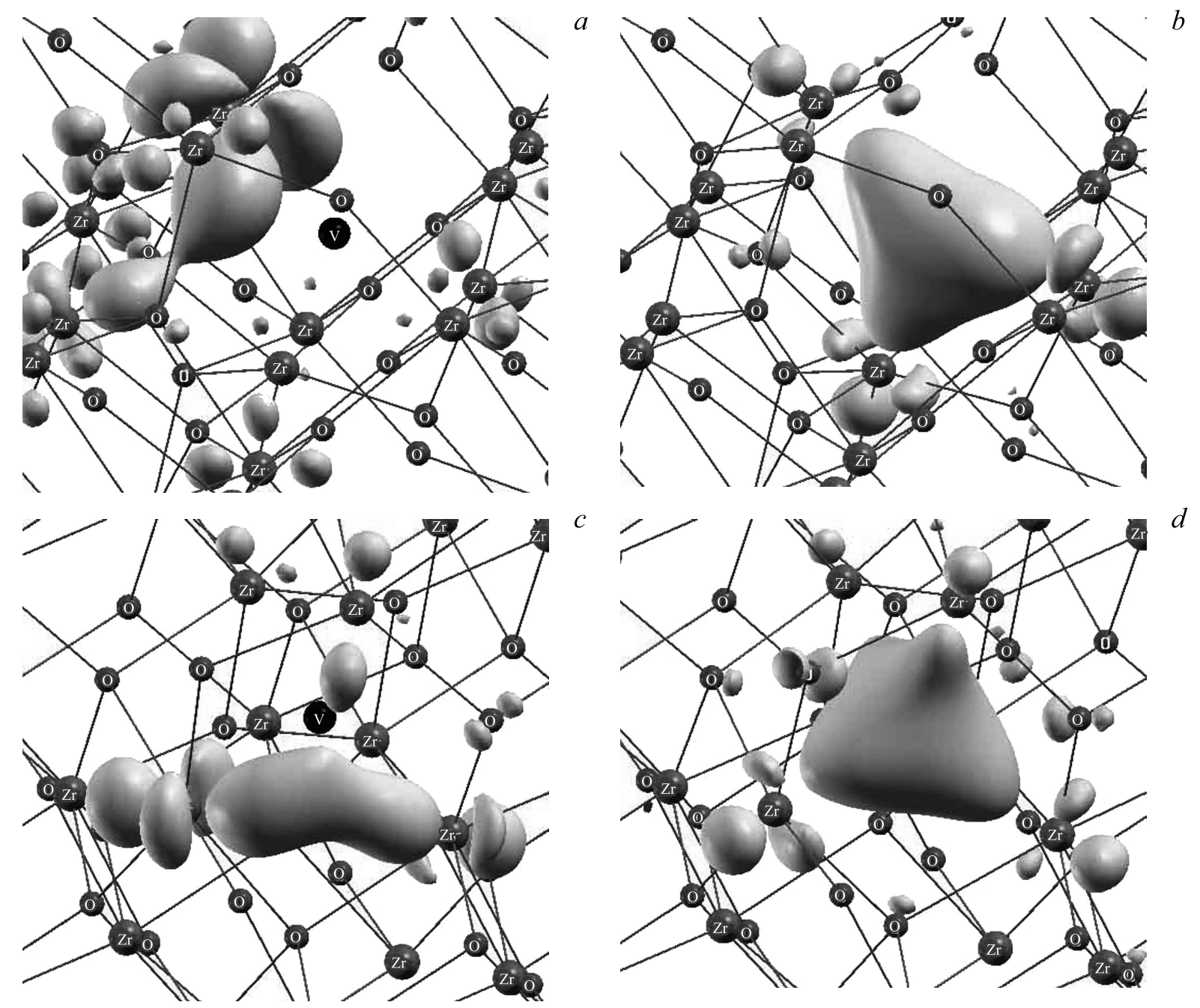

Рис. 2. Изоповерхности равной величины зарядовой плотности в 96 -атомной суперячейке $m-\mathrm{ZrO}_{2}$ с однократно отрицательно $(a, c)$ и положительно $(b, d)$ заряженной вакансией кислорода в трехкратной $(a, b)$ и четырехкратной $(c, d)$ координации. 

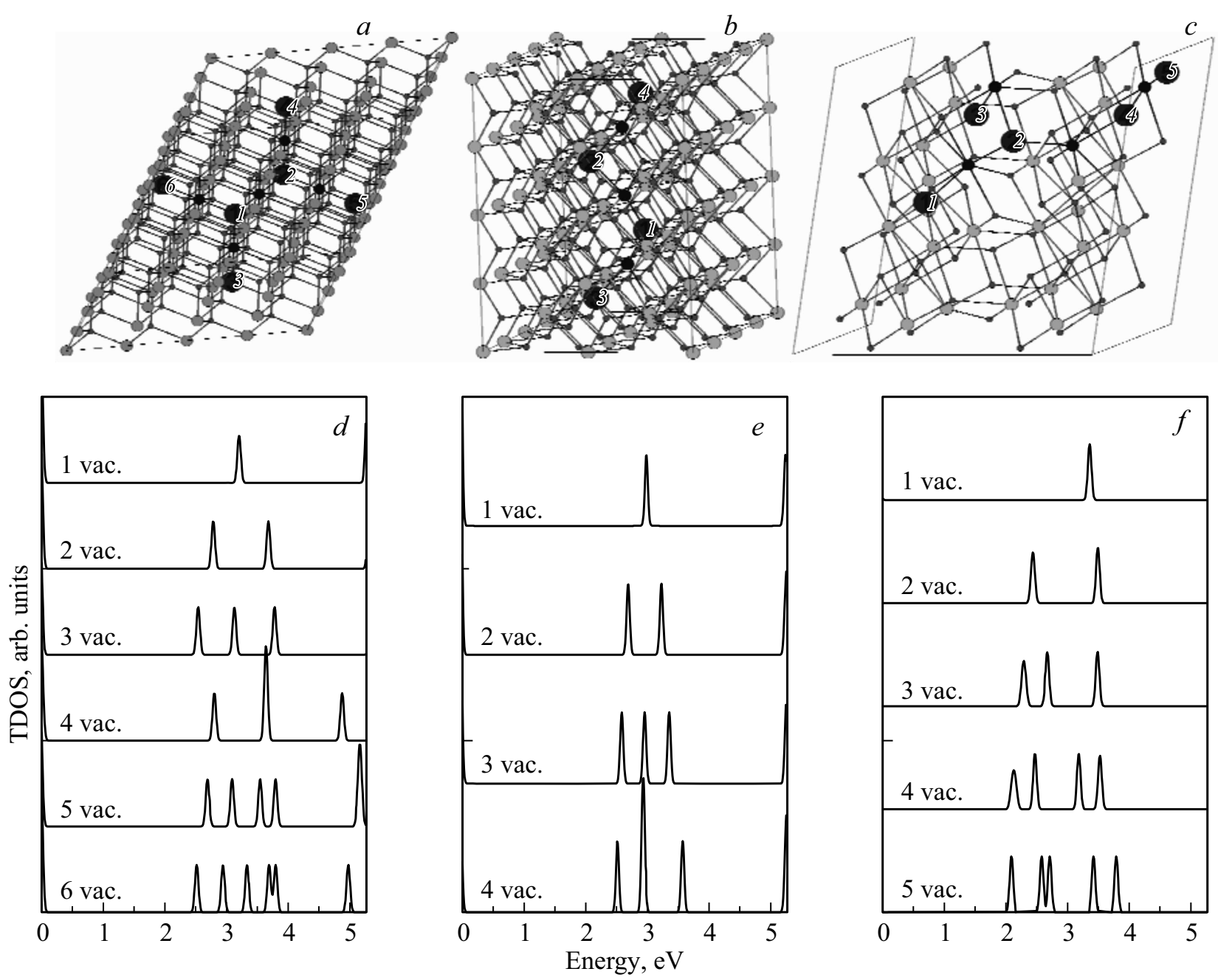

Рис. 3. Суперячейки кристаллов (a) $c$ - (192 атома), (b) $t$ - (192 атома) и (c) $m-\mathrm{ZrO}_{2}$ (96 атомов). Черным цветом обозначены атомы Zr, связанные с парой удаляемых атомов О. Цифрами обозначена последовательность удаления. Фрагменты спектров TDOS, отображающие дефектные состояния в запрещенной зоне, обусловленные наличием поливакансии кислорода в $c$ - $(d), t$ - $(e)$ и $m-\mathrm{ZrO}_{2}(f)$. Нуль энергии соответствует положению $E_{V}$, верхняя граница энергии $-E_{C}$.

представленные данные однозначно демонстрируют, что кислородная вакансия в $m-\mathrm{ZrO}_{2}$ является и электронной, и дырочной ловушкой (является амфотерным дефектом). Следовательно, вакансии $\mathrm{O}$ в $m-\mathrm{ZrO}_{2}$ могут принимать участие в транспорте заряда. Поскольку электронные свойства твердых тел определяются главным образом сортом атомов, можно ожидать, что сделанный вывод справедлив также для аморфных и поликристаллических фаз $\mathrm{ZrO}_{2}$. Значения, представленные в таблице, можно сопоставить с экспериментально определенными величинами $E_{\mathrm{th}}=1.15$ [13] для $\mathrm{ZrO}_{2}$ и $E_{\mathrm{th}}=1.25 \mathrm{eV}$,
$E_{\mathrm{opt}}=2.5 \mathrm{eV}$ для $\mathrm{HfO}_{2}[14]$. Для $V^{0}$ и $V^{+}$имеется хорошее согласие расчетных и экспериментальных данных.

Атомная структура поливакансий кислорода в $c$-, $t$ и $m-\mathrm{ZrO}_{2}$ (рис. 3) показывает, что для каждой последующей вакансии выгодно формирование вблизи уже существующих, причем с одним атомом $\mathrm{Zr}$ связаны не более двух атомов O, подвергающихся удалению. Для $t-\mathrm{ZrO}_{2}$ наблюдается упорядоченность в расположении вакансий в виде одномерной цепочки, ориентированной вдоль кристаллографической оси [001] (причем в отсутствии внешнего поля). Можно предположить, что этот

Значения $E_{\text {opt }}$ и $E_{\text {th }}$ вакансии $\mathrm{O}$ в различных зарядовых состояниях в $m-\mathrm{ZrO}_{2}$

\begin{tabular}{c|c|c|c|c|c}
\hline & $V_{+}$ & $V^{0}$ & $V^{-1}$ & $V^{-2}(s)$ & $V^{-2}(t)$ \\
\hline$E_{\text {opt }}$ (3-fold/4-fold), eV & $2.07 / 2.48$ & $1.71 / 2.15$ & $0.25 / 0.61$ & $0.22 / 0.57$ & $0.21 / 0.35$ \\
$E_{\text {th }}$ (3-fold/4-fold), eV & $1.25 / 1.45$ & $1.15 / 1.50$ & $0.16 / 0.33$ & $0.21 / 0.26$ & $0.21 / 0.26$
\end{tabular}


результат будет справедлив и для аморфной структуры $\mathrm{ZrO}_{2}$, а внешнее электрическое поле будет определять наиболее предпочтительное направление роста цепочки вакансий. Это предположение согласуется с филаментарной моделью резистивного переключения.

Поскольку энергетически выгодно формирование пар близкорасположенных вакансий кислорода (дивакансий), то и в реальных пленках $\mathrm{ZrO}_{2}$, обедненных кислородом, их концентрация должна быть достаточно высока. Отметим, что данные результаты справедливы лишь при условии последовательного роста размера поливакансии (т.е. числа удаленных атомов кислорода).

Каждая последующая вакансия добавляет один заполненный уровень в запрещенную зону $\mathrm{ZrO}_{2}$. Близость вакансий $\mathrm{O}$ приводит к их сильному взаимодействию, что подтверждает смещение дефектных пиков в запрещенной зоне при добавлении каждой последующей вакансии. Уровни от поливакансий $\mathrm{O}$ распределяются с преимущественной локализацией вблизи положения дефектного состояния от моновакансии О. Рост размера поливакансии кислорода формирует в энергетическом спектре $\mathrm{ZrO}_{2}$ широкую заполненную дефектную зону.

Принимая во внимание установленный факт, что для моновакансии кислорода дополнительный электрон распределяется между парой ближайших атомов $\mathrm{Zr}$, т.е. образуется своего рода $\mathrm{Zr}-\mathrm{Zr}$-связь, можно сделать вывод, что при формировании поливакансий кислорода $\mathrm{ZrO}_{2}$ может приобрести металлическую проводимость, которая осуществляется по каналу из ионов $\mathrm{Zr}$, разделяющих вакансии в цепочке.

\section{3. Заключение}

В кубической, тетрагональной и моноклинной фазах $\mathrm{ZrO}_{2}$ положения дефектных состояний в запрещенной зоне, обусловленные вакансией кислорода в различных зарядовых состояниях, а также положительные значения энергии локализации электрона и дырки на вакансии кислорода свидетельствуют о том, что вакансия кислорода является ловушкой как для электрона, так и для дырки. Расчетное положение дефектных уровней в запрещенной зоне, а также величина термической энергии ионизации вакансии кислорода в $m-\mathrm{ZrO}_{2}$ согласуются с известными из литературы экспериментальными данными для аморфного $\mathrm{ZrO}_{2}$. Следовательно, вакансии кислорода являются центром локализации заряда, могут принимать участие в транспорте заряда и отвечать за высокую проводимость диэлектрика.

Для каждой последующей вакансии кислорода энергетически выгодно формирование вблизи уже существующих вакансий, при этом с одним атомом металла связано не более двух атомов кислорода, подвергающихся удалению. Для $t-\mathrm{ZrO}_{2}$ обнаружена упорядоченность вакансий кислорода в виде линейной цепочки. Вакансионные комплексы из $N$ вакансий кислорода формируют в запрещенной зоне $N$ заполненных электронами уровней, причем эти уровни локализуются вблизи уровня моновакансии кислорода.

\section{Список литературы}

[1] X.D. Huang, R.P. Shi, P.T. Lai. Appl. Phys. Lett. 104, 162905 (2014).

[2] C.H. Lai, H.W. Chen, C.Y. Liu. Materials 9, 551 (2016).

[3] I. Karkkanen, A. Shkabko, M. Heikkila, J. Niinisto, M. Ritala, M. Leskela, S. Hoffmann-Eifert, R. Waser. Phys. Status Solidi A 211, 301 (2014).

[4] A.S. Foster, V.B. Sulimov, F.L. Gejo, A.L. Shluger, R.M. Nieminen. Phys. Rev. B 64, 224108 (2001).

[5] J.X. Zheng, G. Ceder, T. Maxisch, W.K. Chim, W.K. Choi. Phys. Rev. B 75, 104112 (2007).

[6] C. Arhammar, C.M. Araujo, R. Ahuja. Phys. Rev. B, 80 115208 (2009).

[7] P.J. Shen, S.P. Jiang, K.P. Ong, W.Z. Ding, P.L. Mao, X.G. Lu, C.H. Li, P. Wu. J. Alloys Compd. 506, 898 (2010).

[8] J.L. Lyons, A. Janotti, C.G. Van de Walle. Microelectron. Eng. 88, 1452 (2011).

[9] C. Gionco, M.C. Paganini, E. Giamello, R. Burgess, C. Di Valentin, G. Pacchioni. Chem Mater. 25, 2243 (2013).

[10] J.P. Holgado, J.P. Espinos, F. Yubero, A. Justo, M. Ocana, J. Benitez, A.R. Gonzalez-Elipe. Thin Solid Films 389, 34 (2001).

[11] D. Muñoz Ramo, J.L. Gavartin, A.L. Shluger, G. Bersuker. Rev. B 75, 205336 (2007).

[12] R.H. French, S.J. Glass, F.S. Ohuchi, Y.N. Xu, W.Y. Ching. Phys. Rev. B 49, 5133 (1994).

[13] G. Jegert, A. Kersch, W. Weinreich, U. Schroder, P. Lugli. Appl. Phys. Lett. 96, 062113 (2010).

[14] V.A. Gritsenko, T.V. Perevalov, D.R. Islamov. Phys. Rep. 613, 1 (2016). 